\title{
Recommendations for standards of sedation and patient monitoring during gastrointestinal endoscopy
}

\author{
G D Bell, R F McCloy, J E Charlton, D Campbell, N A Dent, M W L Gear, R F A Logan, \\ C H J Swan
}

Summary of main recommendations

(1) Safety and monitoring should be part of a quality assurance programme for endoscopy units (section 1.5).

(2) Resuscitation equipment and drugs must be available in the endoscopy and recovery areas (section 2.5).

(3) Staff of all grades and disciplines should be familiar with resuscitation methods and undergo periodic retraining (section 2.6).

(4) Equipment and drugs necessary for the maintenance of airway, breathing, and circulation should be present in the endoscopy unit and recovery area (if outside the unit) and checked regularly (sections 2.3 and 3.2).

(5) A qualified nurse, trained in endoscopic techniques and adequately trained in resuscitation techniques, should monitor the patient's condition during procedures (section 3.1 and 7.1).

(6) Before endoscopy, adverse risk factors should be identified. This may be aided by the use of a check list (sections 4.1 and 4.2).

(7) The dosage of all drugs should be kept to the minimum necessary. There is evidence that benzodiazepine/opioid mixtures are hazardous (section 5.2 and 5.3).

(8) Specific antagonists for benzodiazepines and opioids exist and should be available in the event of emergency (section 5.4).

(9) A cannula should be placed in a vein during endoscopy on 'at risk' patients (section 5.5).

(10) Oxygen enriched air should be given to 'at risk' patients undergoing endoscopic procedures (section 6.2).

(11) The endoscopist should ensure the well being and clinical observation of the patient undergoing endoscopy in conjunction with another individual. This individual should be a qualified nurse trained in endoscopic techniques or another medically qualified practitioner (section 7.1).

British Society of

Gastroenterology, Endoscopy committee working party, St Andrew's Place, London G D Bell

R F McCloy

J E Charlton

D Campbell

NA Dent

$M$ W L Gear

R F A Logan

C H J Swan

Correspondence to:

Dr G D Bell, The Ipswich

Hospital, Heath Road Wing,

Ipswich, Suffolk

Accepted for publication

4 February 199
(12) Monitoring techniques such as pulse oximetry are recommended (section 8.5).

(13) Clinical monitoring of the patient must be continued into the recovery area (section 9.1). (14) Records of management and outcome should be collected and will provide data for appropriate audit (section 10.1).

\section{Introduction}

1.1 Gastrointestinal endoscopy is generally regarded as a safe procedure, but there are many potential hazards. The incidence of serious complications associated with diagnostic upper gastrointestinal endoscopy is about 1 in 1000 procedures.' Mortality is estimated to be between 1 and 6 per 20000 examinations. ${ }^{1-3}$ 1.2 A recent review concluded that the complication rate of upper gastrointestinal endoscopy had decreased by about $40 \%$ over the past 15 years from $0 \cdot 13 \%$ to $0 \cdot 08 \%$. This was accompanied by a slight increase in mortality. ${ }^{3}$ In upper gastrointestinal endoscopy, cardiopulmonary complications predominate while perforation and bleeding are relatively rare. The reverse is true for colonoscopy, where perforation and bleeding are more common. ${ }^{3}$

1.3 There is evidence that adverse cardiopulmonary events may be associated with the use of intravenous drugs. ${ }^{46}$

1.4 The working party seeks to make recommendations that will improve patient safety by minimising the risk of adverse cardiopulmonary events.

1.5 Safety and monitoring should be part of a quality assurance programme for endoscopy units.

\section{Role of the endoscopist}

2.1 Where intravenous sedation techniques are used, experienced endoscopists may assume responsibility for both sedating the patient and performing the endoscopic procedure, provided that adequate assistance is available. It is recommended, at a minimum, that a qualified nurse trained in endoscopic techniques and dedicated to patient care only, is present throughout.

2.2 It is recommended that the consultant responsible for the procedure ensures that the medical staff being trained in endoscopy are instructed adequately in intravenous sedation techniques. When verbal contact with the patient is lost, an anaesthetic has been administered with all the responsibilities that this entails.

2.3 The patient's clinical conditon will be observed throughout the procedure by both the endoscopist and the qualified nurse, as outlined in section $7 \cdot 1$. Additional monitoring equipment may be used at the discretion of the endoscopist. 2.4 It is the responsibility of the consultant endoscopist and the nurse in charge of the area in which endoscopy is carried out, to ensure that the equipment and drugs necessary for resuscitation are available immediately and are regularly checked. Checking procedures should be carried out before each endoscopy list.

2.5 Equipment available in the endoscopy area and the recovery area (if separate) should include the basic drugs and equipment necessary for the maintenance of airway, breathing, and circula- 
tion, as well as a source of oxygen and suction and a tipping trolley.

2.6 Staff of all grades and disciplines should be familiar with resuscitation methods and undergo periodic retraining. Regular practice in simulated emergencies is recommended.

\section{Role of endoscopy assistants}

3.1 The British Society of Gastroenterology recommendations on Staffing of endoscopy units state that two endoscopy assistants, at least one of whom must be a qualified nurse, are required at each endoscopy. The working party endorses the recommendation that the endoscopy assistants have a key role in the safety and monitoring of the patient's comfort and wellbeing throughout the endoscopic procedure. It is recognised that the endoscopist's attention may be focused on the procedure. Where no other medically qualified practitioner is clinically monitoring the patient the onus for this rests with the qualified nurse.

3.2 All endoscopy assistants must be capable of helping the endoscopist in the case of an emergency such as a respiratory or cardiac arrest and adequate training and periodic retraining should be made available. It is the responsibility of both the endoscopist and the nurse in charge of the area to make sure that all equipment for resuscitation is immediately available and regularly checked. Checking procedures should be carried out prior to each endoscopy list.

\section{Patient safety, identification of risk factors} 4.1 Before endoscopy adverse risk factors should be identified. 'At risk' patients include those who can be classified as ASA (American Society of Anesthesiologists) grades III-V (see Appendix I), the elderly, and those with: heart disease, cerebrovascular disease, significant lung disease, liver failure and jaundice, acute gastrointestinal bleeding, anaemia, morbid obesity, and shock.

4.2 Although inpatients undergoing an endoscopy will have been medically checked there may be important risk factors for endoscopy that need to be noted by the endoscopist. A check list of the type used for day case surgery (example in Appendix II, (a) and (b) could be used to identify these factors.

\section{Sedation techniques}

5.1 At present, over $90 \%$ of all upper gastrointestinal endoscopies in the UK are conducted under intravenous sedation, usually with a benzodiazepine. ${ }^{5}$ Despite recent advances, ${ }^{6}$ there is no tradition of formal training in techniques of sedating patients for endoscopic procedures. All practitioners need proper instruction in sedation techniques and refresher courses where indicated. Increased provision of training in sedation should be considered.

5.2 Cardiorespiratory complications are responsible for most deaths after endoscopy. ${ }^{3-6}$ The safety of any endoscopic procedure will be improved by keeping the dosages of all drugs to the minimum amount, permitting patient com- fort and successful performance of the procedure. There can seldom be a reason to exceed the manufacturer's recommended dose schedule ${ }^{7}$ for any sedative drug and then only with great caution. Particular attention should be given to the dose limits for elderly patients and those with coexisting medical diseases such as cardiac, renal, and hepatic failure.

5.3 It is recognised that the combination of a benzodiazepine with an opioid can lead to an increased risk of adverse cardiorespiratory events. ${ }^{58}$ There is evidence that there is a major drug interaction between these two classes of drugs which is synergistic and not simply additive. ${ }^{9}$ If there is a clinical indication to use an analgesic in combination with a benzodiazepine, for example during therapeutic endoscopy, endoscopic retrograde cholangiopancreatography (ERCP), or colonoscopy then the doses of the opioid or benzodiazepine, or both, must be reduced. The opioid should be given first and the benzodiazepine dose titrated carefully with an up to fourfold decrease in total dose. 9

5.4 Specific antagonists for benzodiazepines and opioids exist and should always be available. In the event of an emergency the availability of the antagonists should not encourage any misuse or overdosage of their respective agonists. ${ }^{10}$ Administration of an antagonist does not alter the requirement to maintain vital functions where depression of these has occurred. Flumazenil may take several minutes to reverse completely benzodiazepine induced respiratory depression. The duration of the effect of opioid and benzodiazepine antagonists is less than that of their agonists and reversal may be shortlived.

5.5 A cannula should be placed in a vein during endoscopy on 'at risk' patients. This should become standard practice for all endoscopic procedures.

\section{Preoxygenation and supplemental oxygen}

6.1 Oxygen desaturation occurs frequently during endoscopy, particularly when the procedure is being carried out by inexperienced endoscopists ${ }^{11}$ and when the patient has chronic obstructive airways disease. The provision of oxygen enriched air for the patient to breathe before and during endoscopy has been shown to diminish greatly or prevent hypoxaemia. ${ }^{12} 13$ The amount of supplemental oxygen used (2-4 1/minute) will not normally compromise respiratory function in patients with chronic obstructive airways disease.

6.2 It is recommended that oxygen enriched air be given to 'at risk' patients undergoing endoscopic procedures.

\section{Clinical monitoring}

7.1 The endoscopist should ensure the wellbeing and clinical observation of the patient undergoing endoscopy in conjunction with another individual. This individual should be a qualified nurse trained in endoscopic techniques or another medically qualified practitioner.

7.2 Clinical observation of the early signs of respiratory depression and hypoxia is known to be unreliable. ${ }^{\text {s }}$ It may also be difficult, if not 
impossible, to observe a patient's colour in a darkened room. The use of pulse oximeters (see below) and the prophylactic use of supplemental oxygen is recommended and it is suggested that the endoscopy be carried out in normal lighting.

\section{Continuous monitoring}

8.1 It is known that over $50 \%$ of serious adverse reactions during endoscopy are cardiopulmonary ${ }^{3-5}$ and falls in oxygen saturation occur frequently during upper and lower gastrointestinal endoscopy. ${ }^{46}$ It is thought that during endoscopy most cardiac dysrhythymias occur as a consequence of hypoxia. ${ }^{46}$ Despite these facts the use of oximetry and continuous ECG monitoring remains contentious. ${ }^{4}$

8.2 There is no evidence that the routine use of monitoring equipment will reduce the morbidity and mortality associated with endoscopy, ${ }^{4}$ but the adoption of the following recommendations should encourage high quality patient care. The use of monitoring devices must not distract the endoscopist and assistant from basic clinical observations.

8.3 Pulse oximeters can detect oxygen desaturation that is not apparent clinically and alarm systems may alert the endoscopist in the event of a deterioration in the patient's clinical condition. This may be treated by encouraging the patient to breathe deeply, with supplemental oxygen, termination of the procedure, assisted ventilation, and pharmacological reversal of either the benzodiazepine or the opioid, or both.

8.4 It is suggested that this level of monitoring should be used during procedures: on all 'at risk' patients (see above); which are difficult and prolonged; performed as an emergency; requiring the use of large diameter upper gastrointestinal instruments; performed in a darkened room, such as ERCP; requiring the use of benzodiazepine/opioid combinations.

8.5 The working party suggests that oximetry or continuous ECG monitoring, or both, may improve the sensitivity of clinical monitoring of patients and could reduce the number of adverse cardiopulmonary events encountered during endoscopy.

\section{Patient recovery}

9.1 Clinical monitoring must always be continued into the recovery period and supplemental oxygen and non-invasive monitoring may be needed for occasional patients.

9.2 The endoscopist should give particular instructions for postendoscopy monitoring and care to a qualified trained nurse responsible for the recovery of the patient. For inpatients, instructions should accompany the patient to the ward (example in Appendix III (a)).

9.3 Day cases should be accompanied home by a responsible adult who should be given written instructions as to what to do and who to contract in the event of problems arising (example in Appendix III (b)). Manufacturers of commonly used sedative drugs recommend that patients who have been sedated with an intravenous benzodiazepine should not drive a car, operate machinery, or drink alcohol for up to 24 hours.
This is irrespective of whether their sedation has been reversed with flumazenil.

\section{Records and audit}

10.1 The use of diagnostic and therapeutic endoscopy has increased dramatically in recent years. Advances continue to be made in the development of new procedures, sedative drugs, and sedation techniques. Records should be kept and will include details of management. Appropriate audit of the conduct and outcome of procedures in necessary.

10.2 It is recognised that it will be necessary to revise the recommendations as technology and clinical practice evolve.

\section{Appendix I}

The American Society of Anesthesiologists classification of physical status.

\section{CLASS I}

The patient has no organic, physiological, biochemical, or psychiatric disturbance. The pathological process for which surgery is to be performed is localised and does not entail a systemic disturbance. Examples: a fit patient with an inguinal hernia, a fibroid uterus in an otherwise healthy woman.

\section{CLASS II}

Mild to moderate systemic disturbance caused either by the condition to be treated surgically or by other pathophysiological processes. Examples: non - or only slightly limiting organic heart disease, mild diabetes, essential hypertension, or anaemia. The extremes of age may be included here, even though no discernible systemic disease is present. Extreme obesity and chronic bronchitis may be included in this category.

\section{CLASS III}

Severe systemic disturbance or disease from what ever cause, even though it may not be possible to define the degree of disability with finality. Examples: severely limiting organic heart disease, severe diabetes with vascular complications, moderate to severe degrees of pulmonary insufficiency, angina pectoris, or healed myocardial infarction.

\section{CLASS IV}

Severe systemic disorders that are already life threatening, not always correctable by operation. Examples: patients with organic heart disease showing marked signs of cardiac insufficiency, persistent angina, or active myocarditis, advanced degrees of pulmonary, hepatic, renal, or endocrine insufficiency.

CLASS V

The moribund patient who has little chance of survival but is submitted to operation in despera- 
tion. Examples: the burst abdominal aneurysm with profound shock, major cerebral trauma with rapidly increasing intracranial pressure, massive pulmonary embolus. Most of these patients require operation as a resuscitative measure with little if any anaesthesia.

\section{Appendix II}

(a) Example of a day case surgery check list that has been adapted for use in an endoscopy unit.

\section{PRE-ENDOSCOPY QUESTIONNAIRE}

To be completed by the patient

PLEASE BRING THIS FORM WITH YOU WHEN ATTENDING FOR THE ENDOSCOPY

Your doctor has asked us to carry out an endoscopy examination. (See accompanying explanation.) Before the examination we would be grateful if you would complete the questionnaire below. This questionnaire is designed to help the doctors who will be looking after you. Please indicate answers to the questions about your health in the appropriate box. If any question is difficult to answer please consult your doctor or telephone the endoscopy department: 123-45678, extension XYZ.

\section{Surname:}

First name:

Date of birth:

(1) How would you rate your general health? (please circle)

Excellent Good Fair Poor

(2) Has there been a recent change in your health? (please circle)

Yes No Comment

(3) Do you have or have you ever had any of these problems (please circle)

(a) Heart attack or heart failure

(b) Stroke

(c) Lung problems (eg asthma, pneumonia, emphysema)

(d) Liver problems or hepatitis

(e) High blood pressure

(f) Diabetes

(g) Bleeding problems

(h) Seizures or epilepsy

(i) Rheumatic Fever

(j) Other (please specify)

(4) Please list any medicines you are taking (including ALL prescription and non-prescription drugs, even aspirin and 'the pill').

\begin{tabular}{|c|c|}
\hline $\begin{array}{l}\text { Name of } \\
\text { medicine }\end{array}$ & $\begin{array}{l}\text { Dosage } \\
\text { (amount) }\end{array}$ \\
\hline $\begin{array}{l}\mathrm{a} \ldots \ldots \ldots \ldots \ldots \\
\mathrm{b} \ldots \ldots \ldots \ldots \ldots \ldots\end{array}$ & \\
\hline ……....... & \\
\hline
\end{tabular}

How many times per day

(5) Are you allergic or sensitive to anything, for example medicines or adhesive tape? (please circle) Yes No

If Yes, please list and describe what happened:

(6) Have you, or any of your close relatives had problems with anaesthetics or sedation? (please circle) Yes No If 'yes', what?.

(7) Have you had a recent problem with (please circle) Cold 'Flu Bronchitis laryngitis Sore throat Fever

(8) Do you have any other health problem at present? (please circle)

Yes No If 'yes', what?

\section{Appendix II}

(b) Example of a check list that should be completed by the nurse.

PRE-ENDOSCOPY QUESTIONNAIRE

To be completed by the nurse

Surname...................First name

Date of birth .............. Hospital no

Weight $\ldots \ldots \ldots \ldots \ldots \ldots \ldots \ldots . \mathrm{kg}$
Haemoglobin .............. g/dl
Urinalysis ..................
LMP ..................... (if $x$ rays being used
during procedure)
BP ........................

Procedure planned

Identification bracelets YES/NO

Consent forms signed YES/NO

Fasted

YES/NO Time last

Bowel prep YES/NO

drink/food ...

Jewellery removed YES/NO

Loose or artificial teeth YES/NO

Contact lenses $\quad$ YES/NO

Allergies $\quad$ YES/NO Specify........

Transport/escort home YES/NO

Valuables deposited YES/NO

NURSING REPORT

Signature of nurse.

Date.

Appendix III

(a) Example of written instructions to accompany inpatient back to ward.

\section{CARE OF PATIENTS AFTER ENDOSCOPY}

(1) On return to the ward, the patient should be kept lying on his side and allowed to sleep for up to two hours.

(2) Then, he or she should be asked to sit up and take a sip of water. If the patient can take this without any pain or coughing he may then take a good drink of water.

(3) Any pain or distress or choking must be reported at once to: the medical registrar medical wards; the surgical registrar - surgical wards and nothing further given by mouth until the patient has been seen.

(4) If the water is swallowed satisfactorily, this may be followed by a normal diet.

(5) Some patients complain of a sore throat the next morning. This is quite normal and will pass after 24-48 hours. 
(6) The patient may return to his normal diet after the endoscopy.

(7) The patient must be accompanied home by a responsible adult if discharged within 24 hours of the endoscopic procedure.

(8) The patient must be advised not to drive a car, operate machinery, or drink alcohol until 24 hours have elapsed after the endoscopy.

(b) Example of written instruction given to outpatient and escort on leaving the Endoscopy Unit.

\section{ENDOSCOPY AFTERCARE}

(1) Please do not drive your car or operate any machinery for 24 hours after the endoscopy.

(2) Please do not drink any alcohol for 24 hours.

(3) You may drink any other fluids.

(4) Go home and rest today.

(5) You may have a sore throat for about $24-48$ hours.

NB. Severe pain in the neck, chest, or abdomen should be reported to your doctor at once or telephone the hospital on: 1234567 extension XYZ.

Follow up appointment:

Own GP in 10 days

Outpatients department

This working party report was accepted by the endoscopy section committee of the British Society of Gastroenterology and approved by the council of the British Society of Gastroenterology in December 1990.
Members of the endoscopy committee working party

G D Bell (chairman), The Ipswich Hospital; R F McCloy (secretary), Manchester Royal Infirmary; J E Charlton (Association of Anaesthetists of Great Britain and Ireland), Royal Victoria Infirmary, Newcastle upon Tyne; D Campbell, Shrewsbury Hospital South; N A Dent, Gloucestershire Royal Hospital; $M$ W L Gear, Gloucestershire Royal Hospital; R F A I Queens Medical Centre, Nottingham; $\mathrm{C} H \mathrm{~J}$ Swan City General Queens Medical Centre, Nottingham; $\mathrm{CH}$ J Swan, City General

1 Cotton PB, Williams CB. Practical gastrointestinal endoscopy. 3rd ed. Oxford: Blackwell, 1990: 52 .

2 Carey WD. Indications, contraindications, and complications of upper gastrointestinal endoscopy. In: Sivak M V, ed. Gastroenterologic endoscopy. Philadelphia: W B Saunders,

3 Hart R, Classen M. Complications of diagnostic gastrointestinal endoscopy. Endoscopy 1990; 22: 229-33.

4 Fleischer D. Monitoring the patient receiving conscious sedation for gastrointestinal endoscopy: issues and guidelines. Gastrointest Endosc 1989; 35: 262-6.

5 Daneshmend TK, Bell GD, Logan RFA. Sedation for upper gastrointestinal endoscopy: results of a nationwide survey. Gut 1991; 32: 12-5.

6 Bell GD, Review article: premedication and intravenous sedaton for upper gastrointestinal endoscopy. Aliment sedaton for upper gastrointestinal

7 ABPI. ABPI data sheet compendium. London: Datapharm Publications, 1990.

8 Murray AW, Morran CG, Kenny GNC, Anderson JR. Arterial oxygen saturation during upper gastrointestinal endoscopy: the effects of midazolam/pethidine combination. Gut 1990; 31: $270-3$.

9 Ben-Shlomo I, Abd-El-Khalim H, Ezry J, Zohar S, Tverskoy M. Midazolam acts synergistically with fentanyl for induction of anaesthesia. Br 7 Anaesth 1990; 64: 45-57.

10 Pearson RC, McCloy RF, Morris P, Bardhan KD. Midazolam and flumazenil in gastroenterology. Acta Anaesthesiol Scand 1990; 34 , Suppl 92:21-4.

11 Lavies NG, Creasy MB, Harris K, Hanning CD. Arterial oxygen saturation during upper gastrointestinal endoscopy: oxygen saturation during upper gastrointestinal endoscopy:
influence of sedation and operator experience. $\mathrm{Am} \mathcal{F}$ Gastroinfluence of sedation and
enterol 1988; 83: 618-22.

enterol 1988; 83: 618-22.
12 Bell GD, Brown NS, Morden A, Coady T, Logan RFA. Prevention of hypoxaemia during upper gastrointestinal endoscopy by means of oxygen via nasal cannulae. Lancel 1987; i: 1022-4.

13 Griffin SM, Chung SCS, Leung JWC, Li AKC. Effect of intranasal oxygen on hypoxia and tachycardia during endoscopic cholangiopancreatography. BMF̈ 1990; 300: 83-84. 